\title{
Subependymal giant cell astrocytoma in the absence of tuberous sclerosis complex: case report
}

\author{
Thomas L. Beaumont, MD, PhD, ${ }^{1}$ Jakub Godzik, MD, ${ }^{1}$ Sonika Dahiya, MD, ${ }^{2}$ and \\ Matthew D. Smyth, MD ${ }^{1,3}$ \begin{abstract}
Hospital, St. Louis, Missouri
\end{abstract} \\ Departments of ${ }^{1}$ Neurosurgery and ${ }^{2 N}$ europathology, Washington University School of Medicine, and ${ }^{3}$ St. Louis Children's
}

\begin{abstract}
The authors report the case of a 14-year-old male with a subependymal giant cell astrocytoma (SEGA) that occurred in the absence of tuberous sclerosis complex (TSC). The patient presented with progressive headache and the sudden onset of nausea and vomiting. Neuroimaging revealed an enhancing left ventricular mass located in the region of the foramen of Monro with significant mass effect and midline shift. The lesion had radiographic characteristics of SEGA; however, the diagnosis remained unclear given the absence of clinical features of TSC. The patient underwent grosstotal resection of the tumor with resolution of his symptoms. Although tumor histology was consistent with SEGA, genetic analysis of both germline and tumor DNA revealed no TSC1/2 mutations. Similarly, a comprehensive clinical evaluation failed to reveal any clinical features characteristic of TSC. Few cases of SEGA without clinical or genetic evidence of TSC have been reported. The histogenesis, genetics, and clinical approach to this rare lesion are briefly reviewed.
\end{abstract}

http://thejns.org/doi/abs/10.3171/2015.1.PEDS13146

KEY WORDS mosaicism; subependymal giant cell astrocytoma; SEGA; tuberous sclerosis complex; TSC; oncology

$\mathrm{S}$ UBEPENDYMAL giant cell astrocytoma (SEGA) is the most common central nervous system tumor in patients with tuberous sclerosis complex (TSC), with a prevalence ranging from $5 \%$ to $20 \% .^{1}$ The lesion occurs most commonly in the first 2 decades of life but has been reported as early as 24 weeks' gestation. ${ }^{9}$ Solitary SEGAs occurring in the absence of any other TSC-related lesions have been reported and are believed to result from somatic mosaicism of the TSC gene. ${ }^{11,13}$ Although a SEGA was previously considered a pathognomonic feature of TSC, reports of solitary SEGAs have led to the lesion's redefinition as a "major feature" in the diagnostic criteria of tuberous sclerosis. ${ }^{16}$ It remains unclear whether a solitary SEGA results from de novo mutations at the TSC locus or from somatic mosaicism. ${ }^{4,10,12,13}$ In approximately $20 \%$ of patients with a clinical diagnosis of TSC, no mutations of the TSC1/2 genes can be identified. ${ }^{2}$ Interestingly, mutation-negative patients have less severe disease than patients with mutation-proven TSC. ${ }^{5}$ Here, we describe a case of solitary SEGA in the absence of any clinical features or genetic evidence of TSC.

\section{Case Report}

History

A 14-year-old boy presented with a several-week history of holocephalic headache that became rapidly progressive over 48 hours with associated nausea, emesis, and dizziness. Over the preceding several months, his family had noted behavioral changes, such as impulsivity and inattention, causing difficulty in school. The patient underwent evaluation and was given a diagnosis of attention deficit hyperactivity disorder for which he was prescribed Adderall and clonidine. The patient's medical history was otherwise unremarkable, and there was no family history of central nervous system neoplasia or neurocutaneous syndromes.

\section{Examinations}

The patient's Glasgow Coma Scale score on admission was 15 , and he was fully oriented. His cranial nerves were intact, and no papilledema was seen on direct funduscopic examination. He had full strength throughout his extremi-

ABBREVIATIONS GFAP = glial fibrillary acidic protein; SEGA = subependymal giant cell astrocytoma; TSC = tuberous sclerosis complex.

SUBMITTED March 26, 2013. ACCEPTED January 21, 2015.

INCLUDE WHEN CITING Published online May 15, 2015; DOI: 10.3171/2015.1.PEDS13146.

DISCLOSURE The authors report no conflict of interest concerning the materials or methods used in this study or the findings specified in this paper. 
ties without pronator drift. No cutaneous stigmata of tuberous sclerosis, such as hypomelanotic macules ("ashleaf spots"), shagreen patches, or sebaceous adenomas, were observed.

Neuroimaging demonstrated a cystic, multilobulated enhancing mass within the left lateral ventricle adjacent to the foramen of Monro, associated with contralateral ventriculomegaly. Marked cystic enhancement was seen on postcontrast CT, with obliteration of the left frontal horn of the lateral ventricle and perilesional edema throughout the left frontal lobe (Fig. 1). The lesion was isointense to the brain on T1-weighted MRI, with moderate edema throughout the left frontal lobe on noncontrast T2-weighted sequences (Fig. 2A and B). Postcontrast sequences demonstrated a mixed cystic and solid mass with avid enhancement and extension into the third ventricle (Fig. 2C and $\mathrm{D})$. The radiographic differential diagnosis included SEGA, central neurocytoma, choroid plexus tumor, astrocytoma, and meningioma. ${ }^{11}$

\section{Operation}

The patient underwent stereotactic left frontal craniotomy via a transcortical approach with gross-total excision of the lesion. A septostomy was performed to provide an additional CSF outflow tract given the marked involvement of the left lateral ventricle and foramen of Monro. An external ventricular drain was placed under direct visualization at the conclusion of the procedure. An intraoperative frozen section suggested a primary glioneuronal tumor.

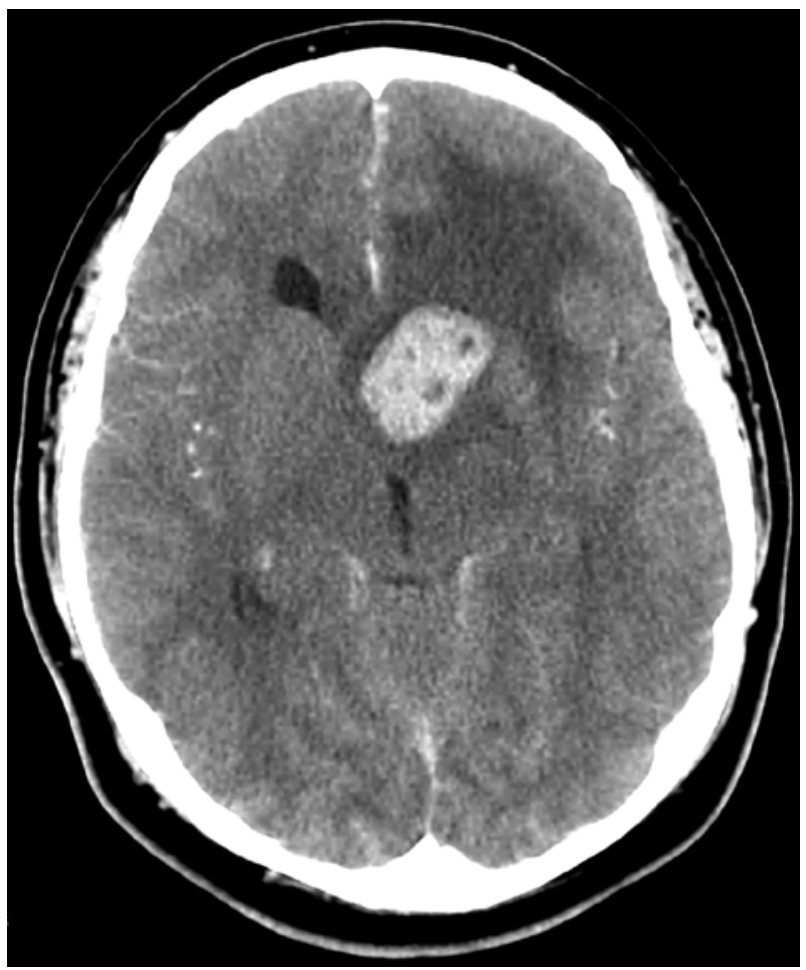

FIG. 1. Preoperative postcontrast axial CT demonstrating a mixed-density cystic mass within the region of the foramen of Monro. There was obliteration of the left frontal horn of the lateral ventricle and extension into the third ventricle. Marked perilesional edema can be seen throughout the left frontal lobe.
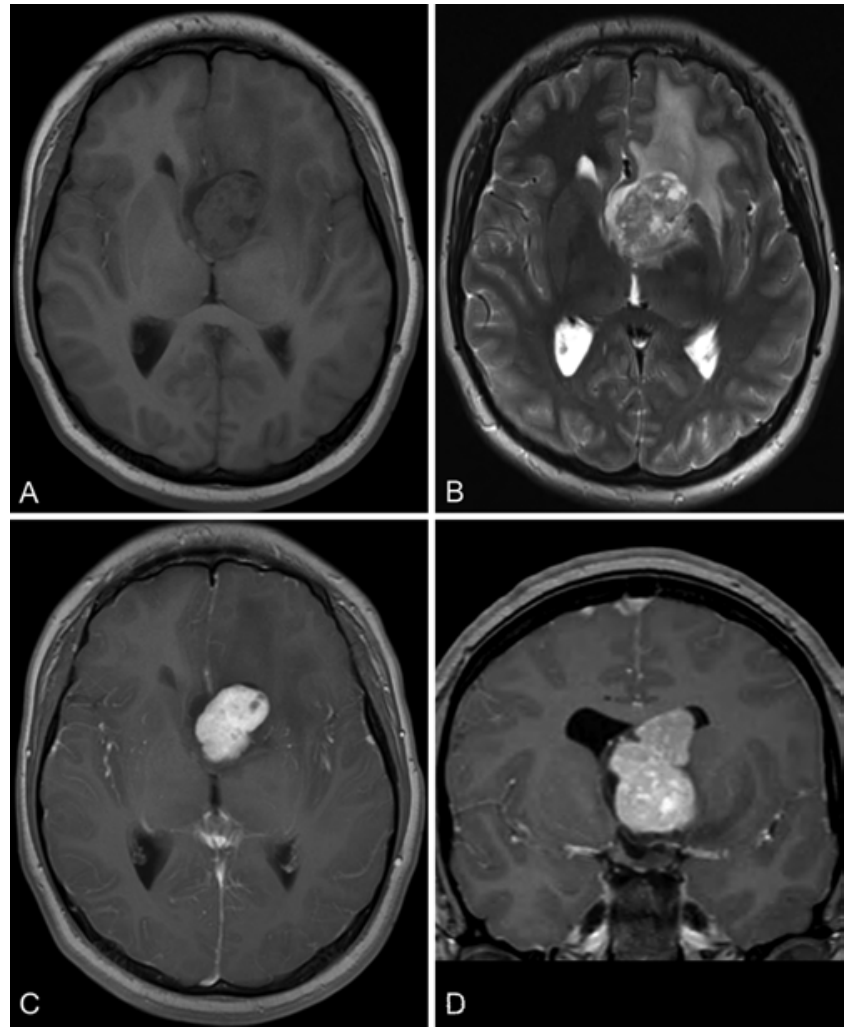

FIG. 2. Preoperative axial noncontrast T1-weighted (A) and T2-weighted (B) MR images demonstrating a well-circumscribed cystic mass within the region of the foramen of Monro with perilesional edema throughout the left frontal lobe. Axial (C) and coronal (D) postcontrast MR images showing obliteration of the left lateral ventricle with avidly enhancing tumor associated with mild ventriculomegaly.

\section{Postoperative Course}

The patient experienced no postoperative complications. His external ventricular drain was weaned without difficulty, and subsequently removed on postoperative Day 5. At the time of discharge from the hospital, the patient's headaches had completely resolved. At his 4-month followup visit, his family described marked improvement in his inattention and impulsiveness. Follow-up neuroimaging at 1 year was without evidence of tumor recurrence or hydrocephalus (Fig. 3). A comprehensive clinical evaluation for TSC was performed including renal ultrasonography, echocardiography, electrocardiography, skeletal imaging, and ophthalmological examination. No features of TSC were identified.

\section{Genetic Analysis}

DNA sequencing of the TSCl and TSC2 genes using peripheral blood failed to show mutations (Athena Diagnostics). At both loci, nonmutant wild-type alleles were observed. Tumor DNA was subsequently isolated from paraffin-embedded tissue and subjected to TSC1 and TSC2 sequencing. The patient was found to have nonmutant sequences in all exons of the TSCl gene, except exons 8 and 16 , which failed to demonstrate polymerase chain reaction products on multiple attempts (Center for Human Genetics). No pathogenic point mutations or deletions were seen in the TSC2 gene. Further analysis of the TSC1 gene was 

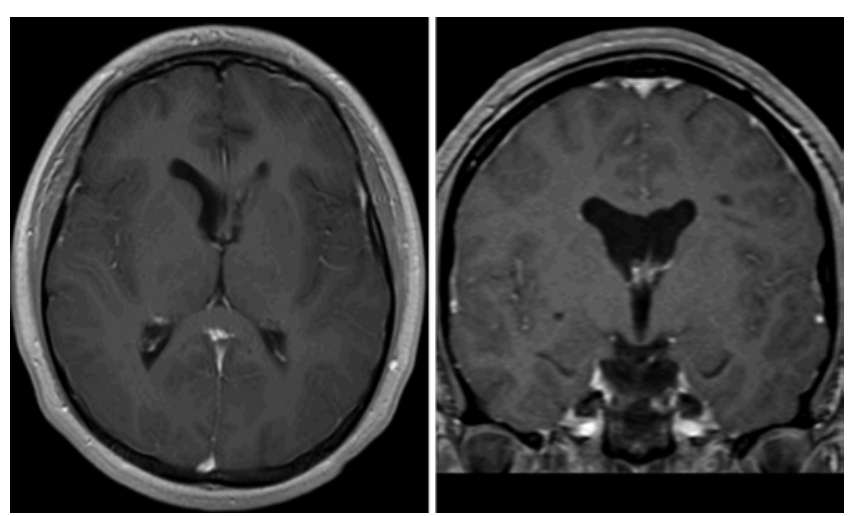

FIG. 3. Postoperative postcontrast axial (left) and coronal (right) T1weighted MR images obtained 4 months postoperatively, showing no evidence of tumor recurrence or progressive ventriculomegaly.

performed using multiple ligation probe amplification to detect whole-exon or whole-gene deletions or duplications. With this method, we confirmed amplification of exons 8 and 16 of the TSC1 gene, and both were nonmutant wild type. This was further confirmed with redesigned sequencing primers. Our analysis was expected to identify $85 \%$ of pathogenic $T S C 1 / 2$ variants but would not detect mosaicism at the TSC locus. Further molecular studies were not possible given the depletion of all tumor specimens.

\section{Pathological Findings}

Histopathological examination of the tumor revealed a low-grade neoplasm comprised of glial cells with phenotypes varying from gemistocytic-like cells, ganglion-like cells, and spindle cells (Fig. 4 left). The cells had moderate to abundant eosinophilic cytoplasm with vesicular nuclei and occasional prominent nucleoli. Immunohistochemically, the cells exhibited faint staining for synaptophysin and were strongly positive for glial fibrillary acidic protein (GFAP) (Fig. 4 right). The neurofilament stain supported a solid growth pattern, while the chromogranin stain was negative. The overall MIB-1 proliferation index was low, reaching $4.8 \%$ in the most active area. Overall findings in conjunction with imaging studies were most consistent with SEGA, WHO Grade I. ${ }^{17,21}$

\section{Discussion}

Subependymal giant cell astrocytoma is a rare central nervous system tumor with mixed glioneuronal features, most frequently seen in the setting of TSC. ${ }^{17}$ Approximately 5\%-20\% of patients with TSC develop SEGA, ${ }^{1}$ although solitary lesions without clinical or radiographic evidence of tuberous sclerosis have been reported.,10,15,17,20,21 It is important to note that none of the previously reported cases were subjected to TSC1/2 gene sequencing, leaving the histogenesis of these particular lesions unclear. Nevertheless, the absence of clinical features of TSC in patients with solitary SEGA has been attributed to somatic mosaicism. ${ }^{13}$ However, both loss of heterozygosity and allelic mutation of TSC 2 have been reported in a single solitary SEGA, suggesting that the genetic etiology may also be the co-occurrence of 2 de novo mutations, as opposed to somatic mosaicism. ${ }^{11}$ In the present case, the patient had a solitary SEGA with neither clinical features of TSC nor a detectable mutation of the TSC1 or TSC2 genes, despite genetic analysis of both germline and tumor using 2 complimentary sequencing methods.

Approximately 38 cases of SEGA in the absence of clinical features of TSC have been described, 7,10,11,15,17-19,21 with the first case reported by Halmagyi and colleagues in 1979. ${ }^{7}$ Bonnin et al. reported on 22 cases of SEGA, and only 5 patients among these cases carried a clinical diagnosis of TSC. ${ }^{3}$ In a cohort of 23 patients with SEGA, clinical manifestations of TSC were identified in only $39 \%$ of the patients during the follow-up period. ${ }^{17}$ Interestingly, 1 patient did not develop symptoms of TSC until 10 years postoperatively. There have been 2 additional reports of SEGA in the absence of TSC. ${ }^{12,20}$ The first included a small case series of 6 patients diagnosed with SEGA, whereas the second identified the disorder at autopsy in a 75-year-old woman without any history or clinical features suggestive of TSC. The diagnoses in these reports were based solely on histopathology, as genetic analysis for the $T S C 1 / 2$ mutations was not performed.

Only 8 patients with solitary SEGA have been studied using molecular or genetic techniques, 7 of whom demonstrated TSC1 or TSC2 germline mutations. Chan et al. ${ }^{4}$ reported $100 \%$ prevalence of $T S C 1 / 2$ mutations in the pe-

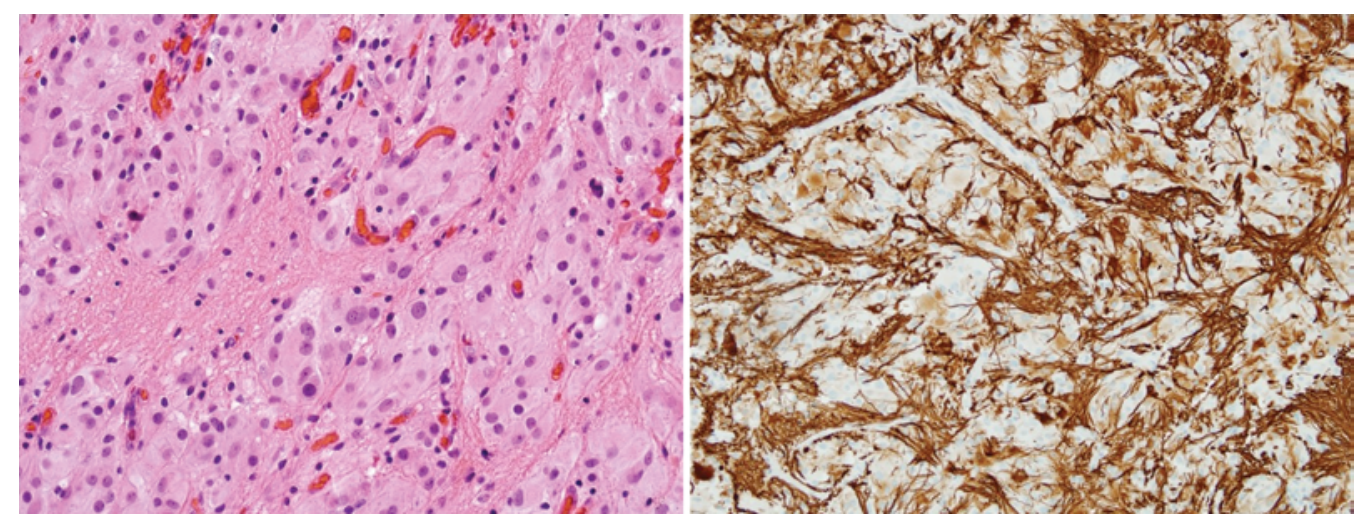

FIG. 4. Left: Photomicrograph of an $\mathrm{H} \&$ E-stained tissue section showing a low-grade glial neoplasm with moderate cellularity and containing a mixture of gemistocytic-like astrocytes, large ganglion-like cells, and spindle cells with fibrillary cytoplasm. Right: Neoplastic cells showing strong and diffuse positivity for GFAP. Original magnification $\times 400$. Figure is available in color online only. 
ripheral blood of 7 patients with a histological diagnosis of SEGA. Notably, 1 patient also demonstrated a somatic TSC2 mutation that was undetectable in the germline (peripheral blood, hair, nails, buccal mucosa). Similarly, there is an additional case report describing a solitary SEGA with an isolated somatic TSC 2 mutation. ${ }^{10}$ In the present case, DNA sequencing of both peripheral blood and tumor tissue failed to reveal mutations or large deletions in the TSC1 or TSC 2 genes. The case represents the third reported instance of the histopathological diagnosis of SEGA in which no mutation of TSC $1 / 2$ could be identified. ${ }^{5}$ Although our genetic analysis was expected to detect at least $85 \%$ of pathogenic TSC1/2 mutations, it is possible we failed to detect novel single-base variants. Furthermore, our study was not designed to detect somatic mosaicism, which has been proposed as an etiology for solitary SEGA. ${ }^{13}$

Subependymal giant cell astrocytoma results from inactivation of the tumor suppressor genes TSC1 on chromosome 9q34 and/or TSC 2 on chromosome 16p13, encoding the proteins tuberin and hamartin, respectively. The tuberin/hamartin complex functions as a suppressor of Ras homolog enriched in brain (RHEB), which directly activates the mammalian target of rapamycin (mTOR). ${ }^{14}$ The complex also inhibits cyclin-dependent kinase inhibitor 1B, which regulates cell cycle progression. Loss of upstream inhibition allows constitutive activation of mTOR and cell cycle progression leading to protein translation, cell growth, and proliferation. ${ }^{6}$ Interestingly, a posttranslational mechanism has been reported, with tuberin inactivated by phosphorylation. ${ }^{8}$ Although not yet explored, a potential epigenetic etiology of SEGA is intriguing to consider, particularly in the setting of a solitary lesion in the absence of features of TSC. Whether by promoter methylation, histone modifications, or nucleosome remodeling, both the solitary cases of SEGA and mutation-negative TSC could result from epigenetic silencing of several tuberin/hamartin complex components. In the present case, the molecular etiology of this solitary SEGA remains unknown.

\section{References}

1. Adriaensen ME, Schaefer-Prokop CM, Stijnen T, Duyndam DA, Zonnenberg BA, Prokop M: Prevalence of subependymal giant cell tumors in patients with tuberous sclerosis and a review of the literature. Eur J Neurol 16:691-696, 2009

2. Au KS, Williams AT, Gambello MJ, Northrup H: Molecular genetic basis of tuberous sclerosis complex: from bench to bedside. J Child Neurol 19:699-709, 2004

3. Bonnin JM, Rubinstein LJ, Papasozomenos SC, Marangos PJ: Subependymal giant cell astrocytoma. Significance and possible cytogenetic implications of an immunohistochemical study. Acta Neuropathol 62:185-193, 1984

4. Chan JA, Zhang H, Roberts PS, Jozwiak S, Wieslawa G, Lewin-Kowalik J, et al: Pathogenesis of tuberous sclerosis subependymal giant cell astrocytomas: biallelic inactivation of TSC1 or TSC2 leads to mTOR activation. J Neuropathol Exp Neurol 63:1236-1242, 2004

5. Dabora SL, Jozwiak S, Franz DN, Roberts PS, Nieto A, Chung J, et al: Mutational analysis in a cohort of 224 tuberous sclerosis patients indicates increased severity of TSC2, compared with TSC1, disease in multiple organs. Am J Hum Genet 68:64-80, 2001

6. Ess KC, Kamp CA, Tu BP, Gutmann DH: Developmental origin of subependymal giant cell astrocytoma in tuberous sclerosis complex. Neurology 64:1446-1449, 2005
7. Halmagyi GM, Bignold LP, Allsop JL: Recurrent subependymal giant-cell astrocytoma in the absence of tuberous sclerosis. Case report. J Neurosurg 50:106-109, 1979

8. Han S, Santos TM, Puga A, Roy J, Thiele EA, McCollin M, et al: Phosphorylation of tuberin as a novel mechanism for somatic inactivation of the tuberous sclerosis complex proteins in brain lesions. Cancer Res 64:812-816, 2004

9. Hussain N, Curran A, Pilling D, Malluci CL, Ladusans EJ, Alfirevic Z, Pizer B: Congenital subependymal giant cell astrocytoma diagnosed on fetal MRI. Arch Dis Child 91:520, 2006

10. Ichikawa T, Wakisaka A, Daido S, Takao S, Tamiya T, Date I, et al: A case of solitary subependymal giant cell astrocytoma: two somatic hits of TSC2 in the tumor, without evidence of somatic mosaicism. J Mol Diagn 7:544-549, 2005

11. Kashiwagi N, Yoshihara W, Shimada N, Tanaka H, Fujita N, Hirabuki N, et al: Solitary subependymal giant cell astrocytoma: case report. Eur J Radiol 33:55-58, 2000

12. Kim S, Lee H, Shin H, Nam D, Kim E, Eoh W: Clinicopathological analysis of subependymal giant cell astocytomas in childhood. J Korean Neurosurg Soc 35:12-16, 2004

13. Kwiatkowska J, Wigowska-Sowinska J, Napierala D, Slomski R, Kwiatkowski DJ: Mosaicism in tuberous sclerosis as a potential cause of the failure of molecular diagnosis. N Engl J Med 340:703-707, 1999

14. Orlova KA, Crino PB: The tuberous sclerosis complex. Ann N Y Acad Sci 1184:87-105, 2010

15. Prahlow JA, Teot LA, Lantz PE, Stanton CA: Sudden death in epilepsy due to an isolated subependymal giant cell astrocytoma of the septum pellucidum. Am J Forensic Med Pathol 16:30-37, 1995

16. Roach ES, Gomez MR, Northrup H: Tuberous sclerosis complex consensus conference: revised clinical diagnostic criteria. J Child Neurol 13:624-628, 1998

17. Sharma MC, Ralte AM, Gaekwad S, Santosh V, Shankar SK, Sarkar C: Subependymal giant cell astrocytoma-a clinicopathological study of 23 cases with special emphasis on histogenesis. Pathol Oncol Res 10:219-224, 2004

18. Shepherd CW, Scheithauer BW, Gomez MR, Altermatt HJ, Katzmann JA: Subependymal giant cell astrocytoma: a clinical, pathological, and flow cytometric study. Neurosurgery 28:864-868, 1991

19. Tabuchi S, Takigawa H, Oka A, Mizuguchi M, Horie Y, Watanabe T: Subependymal giant cell astrocytoma with positive tuberin expression-case report. Neurol Med Chir (Tokyo) 43:616-618, 2003

20. Takei H, Adesina AM, Powell SZ: Solitary subependymal giant cell astrocytoma incidentally found at autopsy in an elderly woman without tuberous sclerosis complex. Neuropathology 29:181-186, 2009

21. Yamamoto K, Yamada K, Nakahara T, Ishihara A, Takaki S, Kochi M, et al: Rapid regrowth of solitary subependymal giant cell astrocytoma-case report. Neurol Med Chir (Tokyo) 42:224-227, 2002

\section{Author Contributions}

Conception and design: Beaumont, Smyth. Acquisition of data: Beaumont, Godzik. Analysis and interpretation of data: all authors. Drafting the article: Beaumont, Godzik. Critically revising the article: Beaumont, Dahiya, Smyth. Reviewed submitted version of manuscript: all authors. Approved the final version of the manuscript on behalf of all authors: Beaumont. Study supervision: Smyth.

\section{Correspondence}

Thomas L. Beaumont, Department of Neurosurgery, Washington University School of Medicine, 660 S. Euclid Ave., Campus Box 8057, St. Louis, MO 63110. email: beaumontt@wustl.edu. 